\title{
Effect of plasmid-mediated RNA interference targeting telomerase reverse transcriptase on lung cancer cells
}

\author{
LINHU GE ${ }^{1-3}$, ZHANSHENG DENG $^{1}$, YANGDE ZHANG ${ }^{1}$, \\ WENLONG SHAO ${ }^{2-4}$, YUAN QIU ${ }^{2,3}$, DONG CUI ${ }^{2,3}$ and DONGHAI HUANG ${ }^{2,3}$ \\ ${ }^{1}$ Department of Surgery, Xiangya Hospital, Central South University, Changsha; ${ }^{2}$ Department of Cardiothoracic \\ Surgery, The First Affiliated Hospital of Guangzhou Medical College, Guangzhou; ${ }^{3}$ Guangzhou Institute \\ of Respiratory Disease and China State Key Laboratory of Respiratory Disease, Guangzhou; \\ ${ }^{4}$ Guangdong Cardiovascular Institute, Southern Medical University, Guangzhou, P.R. China
}

Received June 21, 2011; Accepted July 22, 2011

DOI: 10.3892/or.2011.1455

\begin{abstract}
In the present study, a plasmid-mediated siRNA interference vector targeting the hTERT gene was constructed and stably transfected into H1299 lung cancer cells. Using realtime quantitative fluorescent PCR technology, Western blotting and flow cytometry-based cell cycle profiling, the silencing effect of this vector and its inhibitory effect on proliferation in lung cancer cells were explored. Based upon the results of our previous study, a pair of siRNA sequences was selected, and a DNA template primer was designed and synthesized. After cloning of the template primer into the promoter of the pGenesil-1.1 expression vector, the constructed interference vector was validated using enzyme digestion and gene sequencing. The recombinant interference vector and empty vector were separately transfected into H1299 lung cancer cells with cationic liposomes, and stable monoclonally transfected cells were obtained after selection with G418. After stable transfection, hTERT mRNA and protein expression levels were detected using real-time RT-PCR technology and Western blotting. Using the MTT method and a colony formation assay, the growth and proliferation of the stably transfected lung cancer cells were determined. Changes in the cell cycle profile of the stably transfected lung cancer cells were detected using flow cytometry. An interference vector targeting the hTERT gene (pGenesil.1-hTERT) was successfully constructed. Enzyme digestion and gene sequencing confirmed that the sequence insertion met the criteria of the design. After transfection of H1299 cells with pGenesil.1-hTERT or an empty vector, the stably transfected monoclonal cell lines H1299pGenesil.1-hTERT and H1299-pGenesil.1 were obtained.
\end{abstract}

Correspondence to: Dr Zhansheng Deng, Department of Surgery, Xiangya Hospital, Central South University, 87 Xiangya Road, Changsha 410008, P.R. China

E-mail: drdeng2010@yeah.net

Key words: lung cancer, human telomerase reverse transcriptase gene, RNA interference
Compared to the control cells transfected with the empty vector, the H1299-pGenesil.1-hTERT cells had significantly lower mRNA expression of hTERT $(93.97 \pm 0.83 \%$ inhibition, with $\mathrm{P}<0.001)$. The protein expression of hTERT in H1299pGenesil.1-hTERT cells was significantly lower compared to that in H1299-pGenesil.1 cells. The rate of proliferation of H1299-pGenesil.1-hTERT cells was lower compared to that of H1299-pGenesil.1 lung cancer cells. In H1299-pGenesil.1hTERT cells, the number of cells in the G1 phase increased by $18.3 \%(\mathrm{P}<0.05)$ compared to the control group; the number of cells in the $\mathrm{S}$ and $\mathrm{G} 2$ phases decreased by 10.4 and $7.9 \%$, respectively $(\mathrm{P}<0.05)$. A recombinant plasmid that interfered with the expression of the hTERT target gene was successfully constructed. Upon transfection of the recombinant interference plasmid into H1299 lung cancer cells, hTERT mRNA and protein expression were down-regulated effectively, telomerase activity and cell proliferation were inhibited, and the cell cycle profile was altered.

\section{Introduction}

RNA interference is a gene silencing technology that was developed by Fire et al (1) during their investigation of the mechanism of gene silencing in the nematode $C$. elegans in 1998. When an endogenous mRNA sequence was inserted into a homologous double-stranded RNA in cells, specific degradation of that mRNA occurred, leading to the phenomenon of gene silencing. This gene silencing effect occurred at the post-transcriptional level and is therefore, also known as posttranscriptional gene silencing (PTGS).

The functional mechanism underlying the action of RNAi is a multi-step process that involves many factors induced by double-stranded RNA (dsRNA), is a part of post-transcriptional gene regulation, and requires the participation of ATP. RNA interference includes initiation and effector steps. At the initiation stage, dsRNA is cleaved by the endonuclease RNase III (also known as Dicer in Drosophila) into 21 to 23 nucleotide fragments based on the small interfering RNA (siRNA). At the effector stage, the siRNA binds to Dicer-1 and Dicer-2 (members of the Dicer family), RNAi-specific enzymes (such 
Table I. The primers sequences and lengths of the amplified hTERT and Ipo8 PCR fragments.

\begin{tabular}{lllc}
\hline Gene & & \multicolumn{1}{c}{ Primer sequences } & Length (bp) \\
\hline hTERT & Upstream primer & 5'-GCGTTTGGTGGATGATTTCT-3' & 131 \\
& Downstream primer & 5'-CAGGGCCTCGTCTTCTACAG-3' & \\
Ipo8 & Upstream primer & 5'-AACCAAGGGGTGGTTCATTC-3' & 120 \\
& Downstream primer & 5'-TTGCCACAGCTCTTCATCCT-3' & \\
\hline
\end{tabular}

as AGO-2, MUT-7, RED-1, and PAZ proteins and DNA-RNA helicase) and related proteins to form the RNA-induced silencing complex (RISC). Upon activation by ATP, RISC binds to target mRNA sequences that are homologous to the siRNA. Starting at $12 \mathrm{bp}$ from the 3 ' end of the siRNA, the mRNA is gradually cleaved, resulting in gene silencing (1). At present, the precise mechanism by which the mRNA is cleaved is unclear.

Telomerase is a ribonucleoprotein composed of telomerase RNA (human telomerase RNA, hTR) and protein (2). It is a form of reverse transcriptase that is comprised of three subunits: the RNA component (hTR), the human telomerase reverse transcriptase (hTERT), and telomerase protein 1 (hTEP1) $(3,4)$. Telomerase RNA serves as its own template, allowing reverse transcription to take place continuously at the end of the chromosome in order to synthesize telomere DNA. It compensates for the gradual shortening of the length of telomeres due to mitosis so as to prevent degradation, loss, rearrangement, or confluence of telomere DNA and protect chromosome integrity (5). In healthy humans, telomerase activity can only be detected in germ cells, hematopoietic cells, activated lymphocytes, and other cells with proliferative potential (6). However, telomerase activity can be detected in $90 \%$ of human tumor cells $(3,7)$; moreover, telomerase activity is present in $>80 \%$ of non-small cell lung carcinoma cells $(8,9)$ and is closely related to TNM staging. The more advanced the lung cancer, the higher the telomerase activity $(10,11)$. These data show that the activation of telomerase is a critical step in human carcinogenesis that allows for the maintenance of telomere length. If the telomerase activity present in tumor cells is suppressed, preventing telomere repair, the telomeres will gradually shorten as the cells continue to divide, and the cells will undergo programmed senescence and eventually die. Therefore, telomerase has become a focus for gene-targeted cancer therapy.

As the catalytic subunit of telomerase, hTERT is highly expressed in telomerase-positive tumor tissues and catalyzes the rate-limiting step in telomerase activation, leading to carcinogenesis (12-14). Overexpression of hTERT can result in cell immortalization. Down-regulation of hTERT expression can inhibit telomerase activity, leading to progressively shorter telomeres and effective inhibition of tumor cell growth. Therefore, hTERT may be an ideal target for cancer treatment.

In the present study, a chemically synthesized small hairpin RNA (shRNA) specifically targeting hTERT was cloned into the pGenesil-1.1 plasmid, forming an interference vector. This interference vector was transfected into human lung cancer cells using cationic liposomes, resulting in RNA interference. The investigation of its effect on the expression of the target mRNA and protein, tumor cell proliferation, and cell cycle profile provides experimental evidence of the application of RNA interference technology to the treatment of lung cancer. It is also a new exploration of the significance of telomerase in lung cancer.

\section{Materials and methods}

Materials. The H1299 lung cancer cell line and the E. coli strain DH5a were provided by the National Key Laboratory of Respiratory Diseases. The plasmid pGenesil-1.1 was purchased from Wuhan Jing Sai Biological Engineering Technology Co., Ltd. Primer5 primer software design was used to design the PCR primers for the hTERT gene and the internal reference gene Ipo8; these primers underwent a GenBank Blast homology comparison to determine their specificity and were synthesized by Shanghai Invitrogen Biotechnology Co., Ltd. The primers were diluted to $10 \mu \mathrm{M}$ with RNase-free water before use. The primer sequences and amplified fragment lengths are shown in Table I.

hTERT-siRNA design and synthesis. According to our previous experimental results, a pair of hTERT-siRNA sequences was selected. The siRNA sense strand was 5'-GGA ACACCAAGAAGUUCAU-TT-3'. The antisense strand was 5'-AUGAACUUCUUGGUGUUCC-TT-3'; DNA template primers that incorporated structural features of the plasmid pGenesil-1.1 (Fig. 1) were designed and synthesized.

Based on the afore-mentioned hTERT sense strand corresponding to the cDNA sequence, GGAACACCAAGAAGTT CAT, the primers hTERT-1.1-A (5'-CACCGGAACACCAA GAAGTTCATTTCAAGACGATGAACTTCTTGGTGTTCC TTTTTTG-3') and hTERT-1.1-B (5'-AGCTCAAAAAAGG AACACCAAGAAGTTCATCGTCTTGAAATGAACTTCTT GGTGTTCC-3') were designed. The primer structure of hTERT-1.1-A consisted of the following elements: CACC (underlined)+ Sense + Loop (underlined)+ Antisense + termination signal (underlined)+ SacI site. The DNA template primers were synthesized by Shanghai Invitrogen Biotechnology Co., Ltd.

Cell culture. H1299 human lung cancer cells were cultured in DMEM containing $10 \%$ fetal calf serum, $100 \mathrm{U} / \mathrm{ml}$ penicillin, and $100 \mu \mathrm{g} / \mathrm{ml}$ streptomycin in a $37^{\circ} \mathrm{C}$ incubator with $5 \% \mathrm{CO}_{2}$. In order to maintain cell viability, cells were passaged before they reached $90 \%$ confluency. 


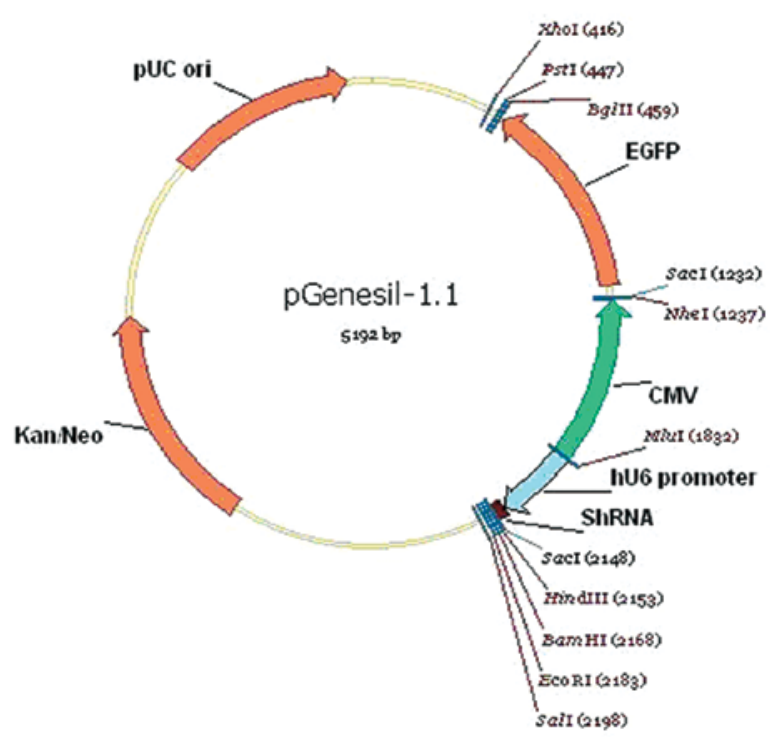

Figure 1. The structural of the plasmid pGenesil-1.1.

Construction of the siRNA expression vector pGenesil.1hTERT. The vector was constructed according to the following procedure. Single-strand target gene fragments were annealed. The single-stranded target genes hTERT-1.1-A and hTERT1.1-B (1 OD each) were added to 30- $\mu \mathrm{l}$ annealing buffer. After mixing in a $94^{\circ} \mathrm{C}$ water bath, reactions were cooled to room temperature. One microliter of annealed product was obtained and added to $99 \mu 1 \mathrm{H}_{2} \mathrm{O}$ to obtain a 100 -fold dilution. The plasmid pGenesil-1.1 was linearized using Eco31I, run on a $1 \%$ agarose gel, and purified from the gel. The annealed fragments of hTERT were ligated into the linearized vector. Five microliters of the ligation preparation was transformed into DH5a competent cells, which were then plated on LB plates containing kanamycin at a final concentration of $30 \mu \mathrm{g} / \mathrm{ml}$. The plates were incubated overnight at $37^{\circ} \mathrm{C}$, and a number of the resultant colonies were inoculated in $3 \mathrm{ml}$ LB medium containing $30 \mu \mathrm{g} / \mathrm{ml}$ kanamycin and grown at $37^{\circ} \mathrm{C}$ with shaking overnight. DNA was prepared according to the Plasmid kit manual and a small amount of plasmid was extracted and analyzed using SacI. The plasmid was also sent to Shanghai Boya Biotechnology Co., Ltd., for sequencing. A large amount of plasmid DNA was prepared according to the large plasmid extraction kit manual and used for subsequent experiments.

Stable transfection of H1299 lung cancer cells. Lung cancer H1299 cells in the logarithmic growth phase were selected. At $24 \mathrm{~h}$ before transfection, the cells were seeded in 6-well culture plates at a density of $1 \times 10^{5}$ cells/well. The cells were transfected the next day at 30-50\% confluency. The cells were transfected with $50 \mathrm{nM}$ plasmid DNA using Lipofectamine 2000 according to the manufacturer's protocol. The cells were incubated for $5 \mathrm{~h}$, observed under a fluorescence microscope and photographed. The transfection medium was then replaced with DMEM containing 20\% fetal bovine serum, and cells were incubated for $48 \mathrm{~h}$. Cells were split 1:3 into 6-well plates in a medium containing $300 \mathrm{mg} / \mathrm{ml} \mathrm{G} 418$. After 11-14 days, the untransfected H1299 cells were completely killed. The
Table II. Reverse transcription reaction reagents.

Final

Reagents

Volume $(\mu 1)$ concentration

\begin{tabular}{lrc}
\hline 5X PrimeScript & \\
& \\
PrimeScript $^{\mathrm{TM}}$ buffer & 4.0 & $1 \mathrm{X}$ \\
Oligo dT primer & 1.0 & \\
Random hexamers & 1.0 & $25 \mathrm{pmol} / \mathrm{l}$ \\
RNase-free $\mathrm{dH}_{2} \mathrm{O}$ & 1.0 & $50 \mathrm{pmol} / \mathrm{l}$ \\
Total RNA $(1000 \mathrm{ng} / \mu \mathrm{l})$ & 12.0 & \\
\end{tabular}

surviving transfected cells were then cultured for 7 to 10 days in medium containing $200 \mathrm{mg} / \mathrm{ml} \mathrm{G} 418$ to obtain resistant clones. Monoclonal cell lines were selected and cultured in a medium containing $200 \mathrm{mg} / \mathrm{ml} \mathrm{G} 418$; they were progressively expanded from 96-well plates up to $75 \mathrm{ml}$ flasks. The two monoclonal stable cell lines used in this study were H1299pGenesil.1-hTERT and H1299-pGenesil.1.

Extraction of total cellular RNA. The culture medium was discarded and the cells were rinsed twice with PBS (pH 7.4). TRIzol ( $1 \mathrm{ml} /$ well) was added and the cells were incubated at room temperature for 5-10 min. The TRIzol extract was placed in a 1.5-ml Eppendorf tube, mixed by inverting the tube 10 times, and incubated at room temperature for 3-5 min. Chloroform $(0.2 \mathrm{ml})$ was added to each tube. Tubes were shaken vigorously by hand for $15 \mathrm{sec}$ and incubated at room temperature for 3-5 min. Tubes were centrifuged at $12,000 \mathrm{x}$ g at $4^{\circ} \mathrm{C}$ for $15 \mathrm{~min}$. The supernatant was then transferred to a new tube. Isopropyl alcohol $(0.5 \mathrm{ml})$ was added to the supernatant. The suspension was mixed and incubated at room temperature for $10 \mathrm{~min}$. Tubes were centrifuged at $12,000 \mathrm{x}$ g at $4^{\circ} \mathrm{C}$ for $10 \mathrm{~min}$. The supernatant was discarded, and $1 \mathrm{ml}$ of $75 \%$ DEPC ethanol was added to precipitate the RNA. Tubes were vortexed and then centrifuged at 7,500 x g at $4^{\circ} \mathrm{C}$ for $5 \mathrm{~min}$. Pellets were washed twice. The supernatant was discarded quickly and carefully. Residual alcohol was removed using a $100 \mu 1$ pipette, and the RNA precipitate was air-dried at room temperature for 5-10 min. RNase-free water was added to dissolve the RNA precipitate, and the solution was incubated for 10 min. One microliter RNase inhibitor was then added to each tube. A small ( $2 \mu \mathrm{l})$ RNA sample was examined by UV spectrophotometry to test its concentration and purity. The optical density ratio (OD 260/280) was in the range 1.8-2.0, indicating relatively pure RNA. In addition, $2 \mu 1$ RNA was run on an agarose gel to check its quality. The extracted RNA was used immediately in the reverse transcription reaction or stored at $-80^{\circ} \mathrm{C}$ for future use.

Reverse transcription reaction. A reverse transcription reaction was performed using $1 \mu \mathrm{g}$ of RNA and a PrimeScript RT Reagent Kit (Takara) according to the manufacturer's instructions. In this reaction, the mRNA was reverse transcribed to cDNA. A $0.2-\mathrm{ml}$ centrifuge tube containing the $20-\mu 1$ reverse transcription reaction was placed on ice (Table II). After the reverse transcription reaction, the cDNA product was stored at $-20^{\circ} \mathrm{C}$ for future use. 
Table III. Real-time-PCR reaction reagents.

Final

Reagents

Volume $(\mu 1)$ concentration

\begin{tabular}{|c|c|c|}
\hline Premix $\operatorname{Ex~Taq}^{\mathrm{TM}}(2 \mathrm{X})$ & 10.0 & $1 \mathrm{X}$ \\
\hline Upstream primers $(10 \mu \mathrm{M})$ & 0.4 & $0.2 \mu \mathrm{mol} / 1$ \\
\hline Downstream primers $(10 \mu \mathrm{M})$ & 0.4 & $0.2 \mu \mathrm{mol} / 1$ \\
\hline cDNA solution & 1.0 & \\
\hline RNase-free $\mathrm{dH}_{2} \mathrm{O}$ & 8.2 & \\
\hline
\end{tabular}

Real time RT-PCR. Either $1 \mu \mathrm{l}$ of the cDNA product or $1 \mu \mathrm{l}$ RNase-free $\mathrm{dH}_{2} \mathrm{O}$ (negative control) was used as template in a SYBR-Green I fluorescence quantitative PCR reaction, allowing detection of the relative expression of hTERT mRNA (Table III). This method yields Ct values for hTERT and Ipo8 (an internal reference gene) expression across all samples. The $\mathrm{Ct}$ value indicates the number of amplification cycles at which the fluorescent signal reaches the set threshold in each reaction tube. The $2^{-\Delta \Delta \mathrm{Ct}}$ method was used to calculate the relative expression of the hTERT gene. This method was designed by Livak and Schmittgen (15) to determine relative gene expression. The relative expression of hTERT mRNA is $2^{-\Delta \Delta \mathrm{Ct}}$, where $\Delta \mathrm{Ct}=\mathrm{Ct}$ (hTERT)-Ct (Ipo8) and $\Delta \Delta \mathrm{Ct}=\Delta \mathrm{Ct}$ (test group) $-\Delta \mathrm{Ct}$ (control group). The percentage inhibition of expression in the silenced cells was calculated using the following formula: inhibition rate $(\%)=(1-$ relative expression of hTERT mRNA in treated cells/relative expression of hTERT mRNA in control cells) x 100\% (16).

Protein extraction. Cells were collected by centrifugation in a $1.5-\mathrm{ml}$ Eppendorf tube. PMSF $(10 \mu \mathrm{l})$ was added to $1 \mathrm{ml}$ RIPA buffer to obtain the lysis buffer. Cells were washed three times with $200 \mu 1$ cold PBS. Cells were lysed in $60 \mu 1$ lysis buffer and lysates were thoroughly mixed before cracking on ice for $30 \mathrm{~min}$. Lysates were centrifuged at $12,000 \mathrm{x}$ g at $4^{\circ} \mathrm{C}$ for $5 \mathrm{~min}$. Supernatants were aliquoted and stored at $-80^{\circ} \mathrm{C}$ for future use.

Determination of the protein concentration. The BCA method was performed using a kit, according to the manufacturer's instructions. A and B solutions were mixed at a 50:1 ratio. Each sample was diluted 6-fold into a final volume of $60 \mu 1$. Each sample ( $25 \mu \mathrm{l}$ was added to each of two duplicate wells. The A:B mixture was then added ( $200 \mu \mathrm{l} /$ well). The plate was incubated at $37^{\circ} \mathrm{C}$ for $30 \mathrm{~min}$. The absorbance at $562 \mathrm{~nm}$ was read using a microplate reader. The standard curve was plotted and the concentrations of the samples were calculated.

SDS-PAGE and Western blotting. Forty micrograms of each sample was added to 5X SDS loading buffer and boiled for 3-5 min. Samples were then run on 12\% SDS-PAGE gels at $100 \mathrm{~V}$ for approximately $1.5 \mathrm{~h}$. Proteins were transferred to PVDF membranes at $30 \mathrm{~mA}$ for $1.5 \mathrm{~h}$. The gels were then stained with Coomassie to ensure complete transfer of the proteins to the PVDF. Membranes were incubated in 5\% skim milk blocking buffer in airtight containers with rocking for $1-2 \mathrm{~h}$ at room temperature or overnight at $4^{\circ} \mathrm{C}$, washed three times for 5 min each in TBST, and incubated in primary antibody (rabbit anti-hTERT polyclonal diluted 1:1000 in 5\% skim milk blocking buffer) with rocking overnight at $4^{\circ} \mathrm{C}$. Membranes were again washed three times for 5 min each in TBST and then incubated in secondary antibody (alkaline phosphataselabeled goat anti-rabbit IgG diluted 1:1000 in 5\% skim milk blocking buffer) with rocking for $2 \mathrm{~h}$ at $4^{\circ} \mathrm{C}$. Membranes were again washed three times for 5 min each in TBST; the excess liquid was then removed using filter paper. LumiGLO chemiluminesence reagent was applied to the membranes for $30 \mathrm{sec}$. The relative amount of hTERT expression was calculated as the raion of the hTERT gray value divided by the gray value of the internal reference protein $\beta$-actin.

Assessment of cell growth using MTT. After trypsin digestion and re-suspension of the cells, the concentration of the suspension was adjusted to 5000 cells $/ \mathrm{ml}$. One hundred microliters of cell suspension was added to each well of a 96-well plate, which was then incubated at $37^{\circ} \mathrm{C}$ with $5 \% \mathrm{CO}_{2}$ for $24-48 \mathrm{~h}$. To each well, $20 \mu \mathrm{l}$ of an MTT solution $(5 \mathrm{mg} / \mathrm{ml}$ or $0.5 \%)$ was added. The plate was then kept in the cell culture incubator for $4 \mathrm{~h}$. The reaction was terminated and the medium was carefully removed from the wells. After addition of $150 \mu \mathrm{l}$ of DMSO to each well, the cell/DMSO/MTT mixtures were transferred to another 96-well plate in order to avoid contamination. The 96-well plate was agitated at a low speed on a shaker for $10 \mathrm{~min}$. The absorbance was then read at $570 \mathrm{~nm}$ on an enzyme-linked immunosorbent assay detector. A control well without cells was used as a zero control. Each sample was tested in triplicate each day for 8 days. The OD values obtained indicate the degree of cell proliferation; from these values, cell growth curves were plotted.

Colony formation assay. After trypsin digestion and re-suspension of cells that were in the logarithmic growth phase, cells were plated at 500 cells/well in a 6 -well plate, which was then incubated at $37^{\circ} \mathrm{C}$ with $5 \% \mathrm{CO}_{2}$ for 1 week. The culture medium was discarded and the cells washed twice with PBS. The cells were then fixed with $2 \mathrm{ml}$ of methanol per well at room temperature for $15 \mathrm{~min}$. The methanol was discarded and the cells stained with $0.4 \%$ crystal violet and rinsed with water. The number of clones per well was counted with the naked eye and the cloning efficiency was calculated using the following formula: Cloning efficiency $(\%)=($ number of colonies formed/number of cells inoculated) $\mathrm{x} 100 \%$.

Flow cytometry-based cell cycle detection. After trypsin digestion and re-suspension of cells in the logarithmic growth phase, cells were washed twice with PBS and digested with the appropriate amount of trypsin. Between $2-3 \times 10^{6}$ cells were collected in a sample tube. Cells were filtered through nylon mesh, washed with PBS, and centrifuged at $1000 \mathrm{rpm}$ for $5 \mathrm{~min}$; the supernatant was discarded. To re-suspend the pellet, $0.2 \mathrm{ml}$ PBS was added dropwise to each tube. Each re-suspension was added dropwise to $5-10 \mathrm{ml}$ of cold $70 \%$ ethanol and fixed overnight at $4^{\circ} \mathrm{C}$. Tubes were centrifuged at $1200 \mathrm{rpm}$ for $10 \mathrm{~min}$ and the supernatant was discarded. The cell pellet was washed 2 or 3 times with PBS. RNase A at a final concentration of $50 \mu \mathrm{g} / \mathrm{ml}$ was added, and the mixture was incubated at $37^{\circ} \mathrm{C}$ for $30-45 \mathrm{~min}$ and cooled on ice for 


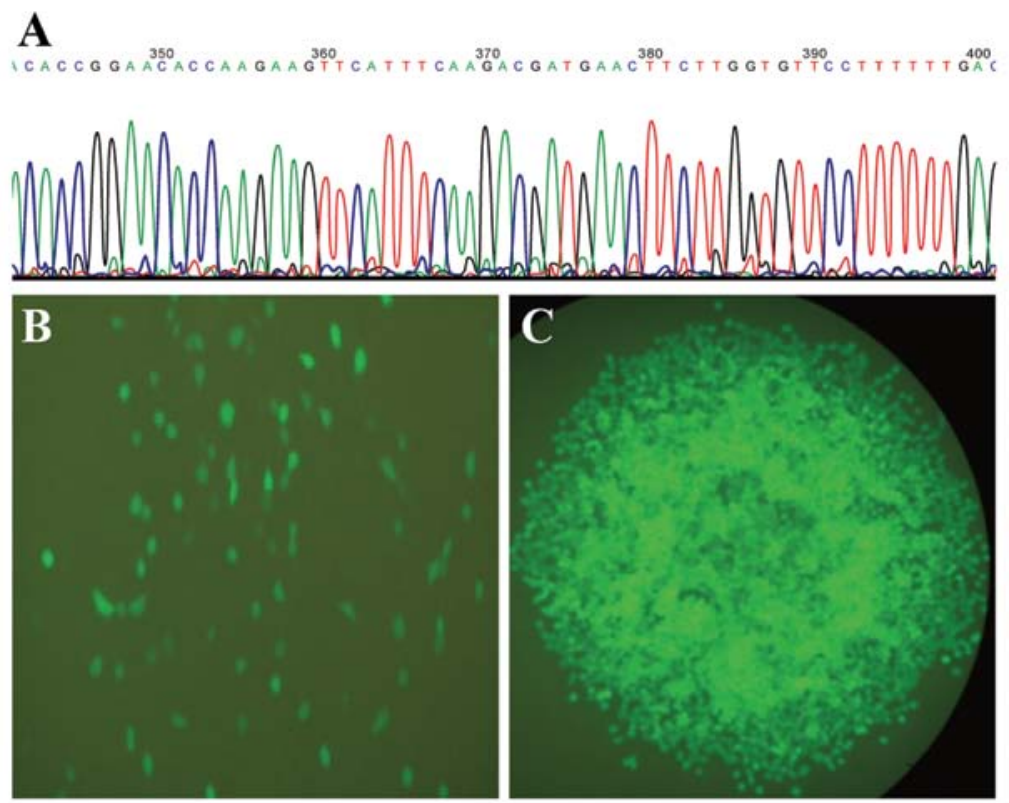

Figure 2. (A) Sequence diagram of pGenesil.1-hTERT; (B) EGFP expression in successfully transfected cells; (C) monoclonal cell colonies.

1 min. Propidium iodide (PI) was added to the cells at a final concentration of $65 \mu \mathrm{g} / \mathrm{ml}$. Cells were stained in the dark at $4^{\circ} \mathrm{C}$ for $30 \mathrm{~min}$. The cell cycle profiles were analyzed using flow cytometry.

Statistical analysis. SPSS15.0 software was used for statistical analysis. Experimental data are presented as means \pm standard deviation $( \pm s)$. If the data displayed a normal distribution and homogeneity of variance, the mean values of two independent samples were compared using a group t-test. Differences with P-values of $<0.05$ were considered statistically significant.

\section{Results}

Observation of H1299 lung cancer cells in culture. The cells were observed under an inverted microscope. H1299 lung cancer cells grew well and appeared to be in good condition. They adhered to the tissue culture dishes and had irregular shapes. Many appeared as long polygonal fusiforms with translucent cytoplasms and indistinct nuclei and could be used for plasmid transfection.

Digestion and sequencing analysis of pGenesil.1-hTERT. The multiple cloning site (MCS) of plasmid pGenesil-1.1 is as follows: -MluI-hU6 promoter-Insert DNA-SacI-. A SacI restriction site was cloned into the target gene fragment to be inserted, and the plasmid pGenesil-1.1 also has a SacI restriction site. SacI digestion of the plasmid with the gene fragment correctly inserted should result in the excision of a $916 \mathrm{bp}$ band. Correct insertion was confirmed by digestion analysis, and the sequencing results accurately reflected the design, as shown in Fig. 2A. Therefore, the pGenesil.1-hTERT vector was successfully constructed.

Transfection of lung cancer cells with the recombinant plasmid. H1299 cells were transfected with the recombinant plasmid pGenesil.1-hTERT or the blank plasmid pGenesil-1.1 using Lipofectamine 2000 for $5 \mathrm{~h}$. About $60 \%$ of the cells expressed EGFP, as observed under the inverted fluorescence microscope (Fig. 2B). G418 (300 mg/ml) was added to selected transfected cells. Within 14 days, the non-transfected cells were all killed. Resistant clones were obtained in culture under G418 selection pressure (Fig. 2C). A number of resistant clones were selected and expanded in cell culture. The stably transfected cell lines H1299-pGenesil.1-hTERT and H1299pGenesil.1 were obtained.

Examination of hTERT $m R$ NA levels in lung cancer cells after stable transfection. The stable transfected cell lines H1299pGenesil.1-hTERT and H1299-pGenesil.1 were selected and lysed for RNA extraction. The extracted mRNA was reverse transcribed into cDNA, and changes in the hTERT mRNA levels were detected using real-time RT-PCR. The mRNA expression of hTERT was significantly decreased in the cells stably transfected with the siRNA plasmid compared with the cells transfected with blank plasmid. The mRNA expression was inhibited by $93.97 \pm 0.83 \%$ a statistically significant difference from the control $(\mathrm{P}<0.01)$ (Fig. 3A).

Analysis of hTERT protein expression by Western blot analysis. The expression levels of hTERT and the reference protein $\beta$-actin in H1299-pGenesil.1-hTERT and H1299pGenesil.1 cells were analyzed by Western blot analysis (Fig. 3B). The intensity of the $\beta$-actin band was almost the same in the RNA interference cell line and in the control, indicating equal loading. According to image analysis, the relative protein expression of hTERT in lung cancer cells transfected with the recombinant plasmid was $0.63823 \pm 0.05448$, and the relative expression of the hTERT protein in lung cancer cells transfected with the blank plasmid was $1.3771 \pm 0.07654$. The difference between the two was statistically significant $(\mathrm{P}<0.01)$, demonstrating that the plasmid encoding RNAi for hTERT was able to suppress hTERT protein expression in H1299 cells. 
$\mathbf{A}$
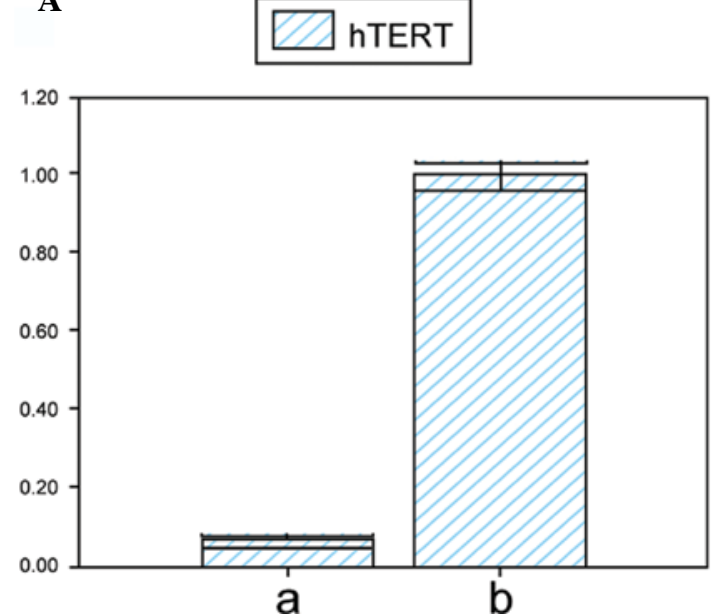

B

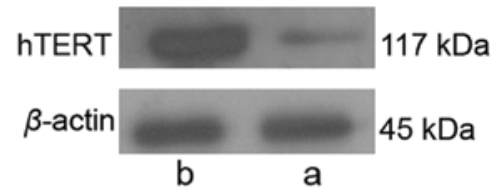

C

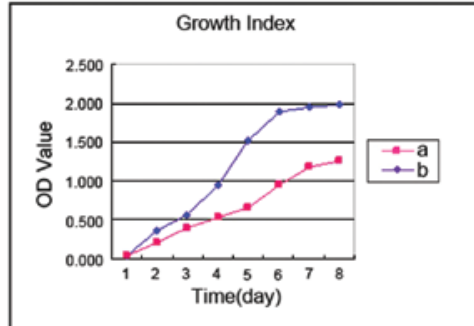

Figure 3. (A) hTERT expression in lung cancer cells transfected with several plasmids; (B) Western blot analysis of hTERT protein expression; (C) cell growth curve. (a) H1299-pGenesil.1-hTERT-1 cells; (b) H1299-pGenesil.1 cells.

A

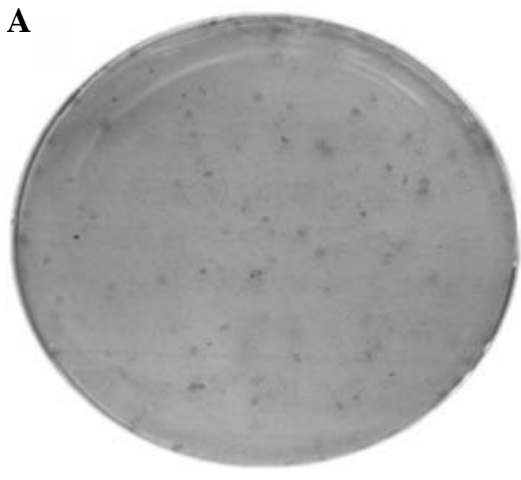

B

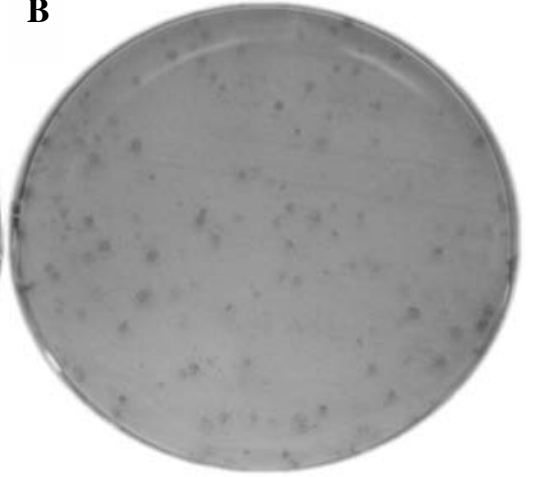

Figure 4. Colony formation assay. (A) H1299-pGenesil.1-hTERT cells; (B) H1299-pGenesil.1 cells.

A

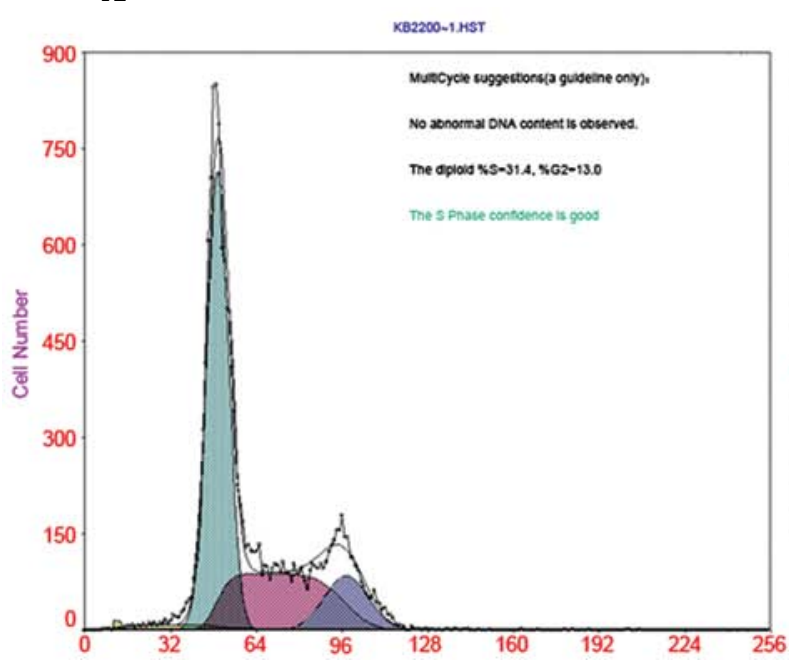

B

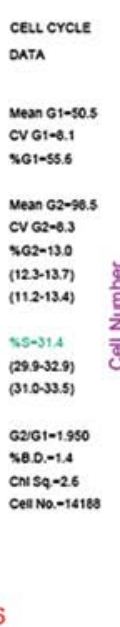

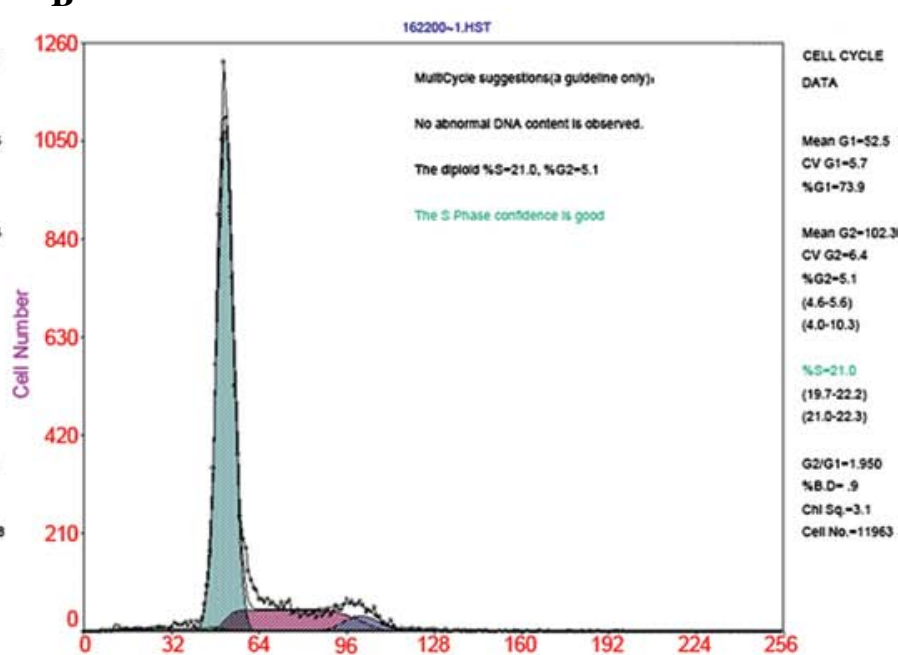

Figure 5. Flow cytometry-based cell cycle detection. (A) Cells transfected with blank plasmid (55.6\% in G1,31.4\% in S, and 13.0\% in G2); (B) cells transfected with recombinant plasmid (73.9\% in G1, $21.0 \%$ in S, and $5.1 \%$ in G2).

Assessment of cell proliferation using the MTT assay. The inhibition of proliferation by pGenesil.1-hTERT was measured in an MTT assay, and the results are shown in Fig. 3C. Lung cancer cells transfected with the recombinant RNAi plasmid had a slower rate of cell proliferation compared to cells transfected with empty plasmid, indicating that the transfection 
of recombinant plasmid could inhibit cell growth and cell proliferation.

Colony formation assay. The results of the colony formation assay are shown in Fig. 4. H1299-pGenesil.1-hTERT cells formed colonies at a rate of $20.45 \pm 0.75 \%$, and $\mathrm{H} 1299$ pGenesil.1 cells formed colonies at a rate of $32.10 \pm 1.46 \%$. The difference between the two was significant $(\mathrm{P}<0.01)$, indicating that the proliferation of the H1299-pGenesil.1-hTERT cells was significantly inhibited.

Cell cycle detection using flow cytometry. The cell cycle profiles of the lung cancer cells H1299-pGenesil.1-hTERT and H1299-pGenesil.1 were ascertained using flow cytometry. The results are shown in Fig. 5. When H1299-pGenesil.1hTERT cells were compared with H1299-pGenesil.1 cells, the percentage of cells in G1 was found to be increased $(18.3 \%$ more than in the control group; $\mathrm{P}<0.05)$, and fewer cells were found to be in $\mathrm{S}$ and $\mathrm{G} 2$ phases (by 10.4 and $7.9 \%$, respectively; $\mathrm{P}<0.05)$. These results suggest that RNAi directed at hTERT arrests the cells in G1 phase, resulting in decreases in cell division and proliferation.

\section{Discussion}

The design of a small interfering RNA plays a crucial role in its effectiveness. The correct and rational design of a siRNA is the key to a successful experiment. When designing an siRNA, attention should be paid to GC content, which should be between $30-70 \%$, with $50 \%$ as the optimum. Care should be taken to avoid homology to other coding sequences and to start at least 100 bases downstream of the start codon. AA dinucleotide sequences and the following 19 to 21 bp should be selected as sites for interference, and a large number of repeats should be avoided, as should 5' and 3 ' non-coding regions and the region around the start codon. These latter areas are rich in regulatory protein binding regions, and the presence of binding proteins or translation initiation complexes may affect the formation of RISC, decreasing the effectiveness of the siRNA. In addition, the $3^{\prime}$ end should be designed to include prominent dTdT or dTdG repeats in order to increase the stability of the siRNA chain and enhance its resistance to degradation by ribozymes, thus extending its length of action (17-19).

There are three methods of small interfering RNA synthesis, including chemical synthesis, in vitro transcription and digestion, and vector-mediated siRNA amplification. Chemical synthesis is the separate synthesis of two single-chains that are then annealed into double-stranded RNA. This method is simple but more expensive. In the in vitro transcription and digestion method, a double-stranded RNA is designed according to the sequence of the target mRNA and its complementary fulllength double-stranded RNA; recombinant Dicer enzyme is then used in vitro to cleave the double-stranded RNA in order to obtain siRNA. Because the sequence of the full-length doublestranded RNA is consistent with that of the target mRNA, a large number of digested siRNA molecules contain a sequence capable of silencing the target gene. This method is cheap, simple, and fast, but it has an obvious disadvantage in that it is likely to cause non-specific gene silencing, particularly of homologous or closely-related genes (19).
Both of these methods still require the siRNA to be introduced into the cell. However, the main drawback of these two methods is that this type of siRNA is quickly degraded and thus has a short duration of action. In light of this problem, plasmid and viral vector-mediated methods of expression of siRNA in vivo have begun to appear. Plasmid-based expression of siRNA requires Pol III promoters located upstream of small hairpin RNA (shRNA) sequences. Plasmid vectors containing a promoter, a shRNA template sequence, and a termination signal can inhibit target gene expression in transfected mammalian cells and thus can be used as 'gene therapy'. The advantage of using plasmid-mediated siRNA is that an siRNA vector can suppress target gene expression for a longer time because of the presence of selectable markers in the siRNA expression plasmid. Since such plasmids can be replicated and amplified, this method can significantly reduce the cost of synthesizing siRNA.

The pGenesil.1 plasmid was selected for the construction of the RNA interference plasmid used in this study. The pGenesil.1 plasmid contains the human U6 promoter, an enhanced green fluorescent protein (EGFP) gene, and a G418 resistance gene. Cells transfected with the EGFP gene expressed green fluorescent protein, which aids in the detection of transfected cells; the G418 resistance gene contributes to the establishment of stably transfected cell lines and the detection of long-term effects of RNAi. When the interference template for the target gene is cloned downstream of the U6 promoter, the inserted sequence is effectively transcribed. Upon encountering the termination signal (composed of 4 to 5 continuous Ts), the transcriptional machinery falls off and transcription terminates immediately, resulting in the completion of the RNA. The $3^{\prime}$ end of the sense strand is connected to the $5^{\prime}$ end of its own anti-sense strand by a man-made hairpin turn and folded into complementary hairpin structures, resulting in a shRNA. Like siRNAs synthesized in vitro, shRNAs can effectively silence their target gene $(20,21)$.

This experiment was based on these principles of siRNA design and our previous experimental results. A pair of siRNA sequences directed against the hTERT gene was chosen, and recombinant plasmid clones were obtained. SacI was used as the restriction enzyme. Since the pGenesil.1 plasmid itself contains a $\mathrm{SacI}$ restriction site, the template insert was designed to have a $S a c$ I restriction site; if the insertion was correct, the plasmid can then be cleaved by SacI into several fragments, including the 916 bp insert. Agarose gel electrophoresis identified a $916 \mathrm{bp}$ band in the digest of the recombinant plasmid by $S a c I$, indicating that the recombinant plasmid pGenesil.1hTERT fulfilled the design requirements, and the sequencing results confirmed the accuracy of the insertion.

The methods of transfection of siRNA into cells include electroporation, calcium phosphate precipitation, microinjection, the DEAE-dextran and polybrene polymer complex method, and the cationic liposome method. Most of these methods are not very efficient, leading to a higher mortality rate, poor reproducibility of experiments, and a narrow range of applications. The cationic liposome method is simple, has many application and high transfection efficiency, and is non-immunogenic. In this study, the cationic liposome Lipofectamine 2000 (obtained from Invitrogen) was selected and used to transfect the plasmids of interest into H1299 lung 
cancer cells. After 5 h, EGFP expression was observed by fluorescence microscopy. The transfection efficiency was above $60 \%$, and no significant changes in cell morphology were seen, indicating a low toxicity. In summary, this reagent provides a fast and practical method of transfection.

Telomerase was discovered by Greider and Blackburn (22) in 1985 in Tetrahymena cell extracts and was subsequently purified. It is a ribonucleoprotein and a form of reverse transcriptase (2). In 1989, the human telomerase was first discovered and identified by Morin (23) in the human cervical cancer cell line HeLa. There are three subunits of human telomerase: the RNA component (hTR), the catalytic subunit of human telomerase reverse transcriptase (hTERT), and telomerase associated protein 1 (hTEP1). In $\sim 90 \%$ of human tumors, regardless of their origin, telomerase activity is present; however, most of the telomerase present in normal tissues is not active (3). Telomerase uses its own RNA as a template; the catalytic subunit (hTERT) uses it repetitively to synthesize telomeres (24). Therefore, telomerase can preserve the length of telomeres. In the absence of telomerase, telomeres gradually shorten, eventually resulting in chromosomal damage; in the presence of telomerase, telomere length will be preserved. This offsets the damage to chromosomes caused by mitosis and the loss of telomere DNA (25). In most human cells, telomerase is not active, resulting in continuous telomere shortening, which ultimately limits cell proliferation and eventually leads to cell death. Telomerase activity can be detected in $90 \%$ of human tumor cells, indicating that activation of telomerase in order to maintain telomere length is a critical step in human carcinogenesis $(7,26)$.

Telomerase is a focus of cancer treatment. Three main telomerase-based methods for treating cancer have been proposed. The first is to directly or indirectly inhibit telomerase activity in order to cause natural shortening of telomeres. The second is to use hTERT as an antigen for immunotherapy, and the third is to target the telomerase promoter, using hTERT as a 'suicide gene'. In all of these proposals, hTERT is seen as the limiting factor for telomerase activity; telomerase activity and hTERT mRNA expression levels are positively correlated. When hTERT is ectopically expressed in telomerase-negative fibroblasts, telomerase activity can be detected, and the cells acquire a tumor cell phenotype (27). Kosciolek et al (28) have used RNAi to specifically inhibit the expression of hTERT, resulting in a $>70 \%$ inhibition in telomerase activity. Other researchers have confirmed that hTERT siRNA inhibits hTERT expression and cell proliferation in liver cancer, breast cancer, laryngeal cancer, and leukemia cells (29). In this study, we chose hTERT as a target for RNA interference in lung cancer cells; the effects of the targeted shRNA on hTERT mRNA and protein expression, cell proliferation, and cell cycle in H1299 lung cancer cells were observed.

H1299 cells were stably transfected with the recombinant RNAi plasmid or a control plasmid, and monoclonal cell lines (termed H1299-pGenesil.1-hTERT and H1299-pGenesil.1) were obtained. mRNA was extracted using TRIzol and reverse transcribed; real-time RT-PCR was used to examine the level of hTERT mRNA expression, which we found to be decreased by at least $93.97 \%$ in the pGenesil.1-hTERT transfected lines compared to controls. Protein expression of hTERT was also decreased. The mechanism of this persistent decrease in hTERT expression could be due to the shRNA-mediated formation of hairpin loops described above, as well as the continuous replication of the plasmid in mammalian cells.

The persistent expression of the RNAi plasmid led not only to a decrease in hTERT expression but also to the inhibition of cell growth and proliferation, as detected using MTT and colony formation assays. We hypothesize that a decrease in hTERT expression leads to a decrease of telomerase activity, progressive shortening of telomeres, and effective suppression of tumor cell growth. These results confirm that the expression of hTERT, which is a suitable target for RNAi, is required for telomerase activity.

The cell cycle refers to the progression of the cell through one round of cell division. Each cell cycle is divided into four highly regulated, sequential phases, G1 (Gap1), S (DNA Synthesis), G2 (Gap 2) and M (Mitosis). Progression through the cell cycle involves many factors, including Myc, which activates the cyclin E/cyclin-dependent kinase 2 (CDK2) and helps to mediate the transition from G1 phase to $\mathrm{S}$ phase (30). At the G1/S checkpoint, levels of cyclin D1 and cyclin E are high, while expression of p16INK4 is low, leading to $\mathrm{Rb}$ phosphorylation. When $\mathrm{Rb}$ is phosphorylated, it is unable to interact with E2F transcription factors, and therefore E2F-1 accumulates in the nucleus, activating DNA replication and the transcription of many cell cycle-related genes. This leads to progression into the $\mathrm{S}$ phase (31). Studies have indicated that telomerase activity is correlated with high expression of cyclin D and low expression of p16INK4 (32-34). In the present study, inhibition of hTERT expression significantly altered the cell cycle profile of H1299 cells; the proportion of cells in G1 was increased (to $73.9 \%$ ), while the percentage of cells in $\mathrm{S}$ and G2 phases was decreased. Thus, upon inhibition of telomerase activity using hTERT RNAi, cyclin D levels may decrease and levels of p16INK4 increase, leading to $\mathrm{G} 1$ arrest via the $\mathrm{Rb}$ pathway described above. However, the exact mechanism by which hTERT RNAi leads to cell cycle arrest is unknown and requires additional studies.

In this study, we used an RNA interference vector to target the hTERT gene in lung cancer cells; the results showed that the vector effectively suppressed the expression of the hTERT gene, altered the cell cycle profile, and inhibited cell proliferation. Interestingly, according to the studies of Sozzi et al (35) and Paci et al (36), hTERT DNA was present at significantly higher levels in the plasma of patients with lung cancer than in the plasma from healthy volunteers, indicating that hTERT is not only an ideal target for gene therapy in lung cancer but could also be used as a diagnostic marker in the blood.

\section{Acknowledgements}

The study was supported by the Guangdong Natural Science Foundation no. 5002320.

\section{References}

1. Fire A, Xu S, Montgomery MK, et al: Potent and specific genetic interference by double-stranded RNA in Caenorhabditis elegans. Nature 391: 806-811, 1998.

2. Greider CW and Blackburn EH: The telomere terminal transferase of Tetrahymena is a ribonucleoprotein enzyme with two kinds of primer specificity. Cell 51: 887-898, 1987. 
3. Kim NW, Piatyszek MA, Prowse KR, et al: Specific association of human telomerase activity with immortal cells and cancer. Science 266: 2011-2015, 1994.

4. Nakamura TM, Morin GB, Chapman KB, et al: Telomerase catalytic subunit homologs from fission yeast and human. Science 277: 955-959, 1997.

5. Raynaud CM, Sabatier L, Philipot O, et al: Telomere length, telomeric proteins and genomic instability during the multistep carcinogenic process. Crit Rev Oncol Hematol 66: 99-117, 2008.

6. Djojosubroto MW, Choi YS, Lee HW, et al: Telomeres and telomerase in aging, regeneration and cancer. Mol Cells 15: 164-175, 2003

7. Wright WE, Piatyszek MA, Rainey WE, et al: Telomerase activity in human germline and embryonic tissues and cells. Dev Genet 18: 173-179, 1996.

8. Taga S, Osaki T, Ohgami A, et al: Prognostic impact of telomerase activity in non-small cell lung cancers. Ann Surg 230: 715-720, 1999

9. Shao WL, Wang DY and He JX: The role of gene expression profiling in early-stage non-small cell lung cancer. J Thorac Dis 2: 89-99, 2010.

10. Hsu CP, Miaw J, Hsia JY, et al: Concordant expression of the telomerase-associated genes in non-small cell lung cancer. Eur J Surg Oncol 29: 594-599, 2003.

11. Xiao DK and He JX: Epithelial mesenchymal transition and lung cancer. J Thorac Dis 2: 154-159, 2010.

12. Elenitoba-Johnson KS: Complex regulation of telomerase activity: implications for cancer therapy. Am J Pathol 159: 405-410, 2001.

13. Majumdar AS, Hughes DE, Lichtsteiner SP, et al: The telomerase reverse transcriptase promoter drives efficacious tumor suicide gene therapy while preventing hepatotoxicity encountered with constitutive promoters. Gene Ther 8: 568-578, 2001.

14. Cukusić A, Skrobot Vidacek N, et al: Telomerase regulation at the crossroads of cell fate. Cytogenet Genome Res 122: 263-272, 2008

15. Livak KJ and Schmittgen TD: Analysis of relative gene expression data using real-time quantitative PCR and the 2(-Delta Delta C(T)) method. Methods 25: 402-408, 2001

16. Stratford S, Stec S, Jadhav V, et al: Examination of real-time polymerase chain reaction methods for the detection and quantification of modified siRNA. Anal Biochem 379: 96-104, 2008

17. Elbashir SM, Martinez J, Patkaniowska A, et al: Functional anatomy of siRNAs for mediating efficient RNAi in Drosophila melanogaster embryo lysate. EMBO J 20: 6877-6888, 2001.

18. Reynolds A, Leake D, Boese Q, et al: Rational siRNA design for RNA interference. Nat Biotechnol 22: 326-330, 2004

19. Ui-Tei K, Naito Y, Takahashi F, et al: Guidelines for the selection of highly effective siRNA sequences for mammalian and chick RNA interference. Nucleic Acids Res 32: 936-948, 2004.
20. Wadhwa R, Kaul SC, Miyagishi M, et al: Vectors for RNA interference. Curr Opin Mol Ther 6: 367-372, 2004

21. Paul CP, Good PD, Winer I, et al: Effective expression of small interfering RNA in human cells. Nat Biotechnol 20: 505-508, 2002.

22. Greider CW and Blackburn EH: Identification of a specific telomere terminal transferase activity in Tetrahymena extracts. Cell 43: 405-413, 1985.

23. Morin GB: The human telomere terminal transferase enzyme is a ribonucleoprotein that synthesizes TTAGGG repeats. Cell 59: $521-529,1989$.

24. Greider CW and Blackburn EH: A telomeric sequence in the RNA of Tetrahymena telomerase required for telomere repeat synthesis. Nature 337: 331-337, 1989.

25. Blackburn EH: Switching and signaling at the telomere. Cell 106 661-673, 2001.

26. Kim NW, Piatyszek MA, Prowse KR, et al: Specific association of human telomerase activity with immortal cells and cancer. Science 266: 2011-2015, 1994.

27. Hahn WC, Counter CM, Lundberg AS, et al: Creation of human tumour cells with defined genetic elements. Nature 400: 464-468, 1999.

28. Kosciolek BA, Kalantidis K, Tabler M, et al: Inhibition of telomerase activity in human cancer cells by RNA interference. Mol Cancer Ther 2: 209-216, 2003.

29. Zhou X and Zhang PH: Stable inhibition of hTERT gene by siRNA in hepatocarcinoma cells. J Fourth Mil Med Univ 26: 2233-2236, 2005

30. Bouchard C, Staller P and Eilers M: Control of cell proliferation by Myc. Trends Cell Biol 8: 202-206, 1998.

31. Dyson N: The regulation of E2F by pRB-family proteins. Genes Dev 12: 2245-2262, 1998 .

32. Soria JC, Morat L, Commo F, et al: Telomerase activation cooperates with inactivation of p16 in early head and neck tumorigenesis. Br J Cancer 84: 504-511, 2001.

33. Henderson YC, Breau RL, Liu TJ, et al: Telomerase activity in head and neck tumors after introduction of wild-type p53, p21, p16, and E2F-1 genes by means of recombinant adenovirus. Head Neck 22: 347-354, 2000

34. Nomoto K, Maekawa M, Sugano K, et al: Methylation status and expression of human telomerase reverse transcriptase mRNA in relation to hypermethylation of the p16 gene in colorectal cancers as analyzed by bisulfite PCR-SSCP. Jpn J Clin Oncol 32: 3-8, 2002.

35. Sozzi G, Conte D, Leon M, et al: Quantification of free circulating DNA as a diagnostic marker in lung cancer. J Clin Oncol 21: 3902-3908, 2003

36. Paci M, Maramotti S, Bellesia E, et al: Circulating plasma DNA as diagnostic biomarker in non-small cell lung cancer. Lung Cancer 64: 92-97, 2009. 